\title{
Direct observation of miniband-edge singularities in the optical spectra of GaAs/AlAs superlattices
}

\author{
Kenzo Fujiwara \\ Kyushu Institute of Technology, Tobata, Kitakyushu 804, Japan \\ Kenji Kawashima and Teiji Yamamoto \\ ATR Optical and Radio Communications Research Laboratory, Soraku-gun, Kyoto 619-02, Japan \\ Naokatsu Sano \\ Kwanseigakuin University, Physics Department, Nishinomiya 662, Japan \\ Roberto Cingolani \\ University of Lecce, Materials Science Department, I-73100 Lecce, Italy \\ Holger T. Grahn and Klaus Ploog \\ Paul-Drude-Institut für Festkörperelektronik, D-10117 Berlin, Germany \\ (Received 6 August 1993; revised manuscript received 12 October 1993)
}

\begin{abstract}
Band-edge optical transitions in GaAs/AlAs superlattices with different miniband widths are investigated by low-temperature-photocurrent, photoluminescence-excitation, and reflectance spectroscopy. Direct spectroscopic evidence is given for excitonic effects at saddle-type critical points which are related to $M_{1}$ Van Hove singularities at the upper miniband edges, for both heavy-hole and light-hole excitons. Excellent agreement is obtained between the observed transition energies and the miniband widths deduced from Kronig-Penney model calculations.
\end{abstract}

Optical transitions in semiconductor superlattices (SL's) have received much attention recently. ${ }^{1-5}$ One of the interesting properties of SL's compared to quantum wells (QW's) is the tunability of the miniband width by varying the composition and thickness parameters. As a result, quantum-mechanical tunneling of the electron wave function can be controlled. ${ }^{6}$ This provides a unique opportunity to systematically study fundamental edge optical transitions in semiconductor SL's with designed miniband widths. For example, using GaAs/AlAs short-period superlattice systems, such miniband structures can easily be realized, and we are able to obtain a joint optical-transition miniband width $2 \Delta$ between 10 and $200 \mathrm{meV}$, which is a sum of the conduction $(2 \Delta e)$ and valence $(2 \Delta h)$ miniband width. In the report by $\mathrm{Chu}$ and Chang, ${ }^{7}$ the saddle-point exciton was predicted to appear in the SL.

In a typical GaAs/AlAs SL, two different types of excitonic effects should exist. In addition to the lower bandedge $M_{0}$ Van Hove singularity, which is even present in bulk semiconductors, the critical point $M_{1}$ at the miniband top, which is formed by zone folding along the $z$ direction, should be observable. However, previous experimental efforts ${ }^{8,9}$ to detect excitonic effects at $M_{1}$ saddle-type critical points ${ }^{10}$ were not very successful. Only faint structures were observed in the photoluminescence excitation (PLE) spectra, because only a narrow range of miniband widths $(\sim 30 \mathrm{meV})$ was investigated and a systematic change of SL parameters was missing. In fact, diamagnetic shifts ${ }^{9}$ had to be employed to identify the upper and lower band edges, hence giving only indirect evidence. As a consequence, no direct spectro- scopic evidence has yet been published for the upper miniband-edge singularity.

In this paper, band-edge optical transitions are investigated in GaAs/AlAs SL's with different miniband widths by employing photocurrent (PC), PLE, and reflectance spectroscopy. By virtue of systematic parameter variations in SL samples of excellent quality, direct spectroscopic evidence is given for the upper miniband-edge singularities and their excitonic effects. A good correlation is obtained between observed and calculated optical transition energies using a simple Kronig-Penney model.

Two different series of high-quality GaAs/AlAs SL samples were grown on $\mathrm{GaAs}(100)$ substrates by molecular-beam epitaxy. The first series of SL samples for PC measurements consisted of $p-i-n$ diode structures, in which the nominally undoped SL is contained in the intrinsic region. ${ }^{11}$ In this series of $12 \mathrm{SL}$ samples, which were grown within a short experimental period to ensure reproducibility of sample quality, the well $\left(L_{z}\right)$ and barrier $\left(L_{B}\right)$ thickness parameters were chosen to form a matrix covering $\left(L_{Z}, L_{B}\right)$ values of $26.1-62.6$ and 5.7-17.3 $\AA$, respectively. The SL period was measured by smallangle $x$-ray-diffraction experiments. These results were used to determine the actual growth rate, from which the nominal values of the well and barrier thickness were estimated. The 100-period SL was confined between $\mathrm{Al}_{0.4} \mathrm{Ga}_{0.6} \mathrm{As}$ cladding layers. The second series consisted of similar SL samples except that all samples for PLE measurements were grown nominally undoped. The well width was precisely fixed at $L_{Z}=24$ monolayers $(67.9 \AA)$ with variations of $L_{B}$ from 1 to 36 monolayers in 
different samples. In this case the growth was controlled by reflection high-energy electron-diffraction (RHEED) intensity oscillations. The PC experiments were performed around $\sim 16 \mathrm{~K}$ in a closed-loop He cryostat using a combination of a tungsten-halogen lamp and a monochromator for excitation and a standard computercontrolled electrometer for dc detection. The PLE and reflectance measurements were made at $4 \mathrm{~K}$ also using a lamp system for excitation and a single photon counting system for detection.

Figure 1 shows typical PC spectra of three SL diode structures with $\left(L_{Z}, L_{B}\right)$ values of (a) $(62.6$ and $5.7 \AA)$, (b) (62.6 and $8.6 \AA$ ), and (c) (39.2 and $8.6 \AA$ ) to illustrate the spectral changes with a variation of both $L_{B}$ and $L_{Z}$. The spectra depicted by solid lines were measured near flatband conditions (under forward bias voltages). In the PC spectra, sharp absorption thresholds are observed. When the AlAs barrier thickness increases, the threshold wavelength (energy) decreases (increases) from spectra (a) to (b). At the absorption edge a sharp peak (indicated by

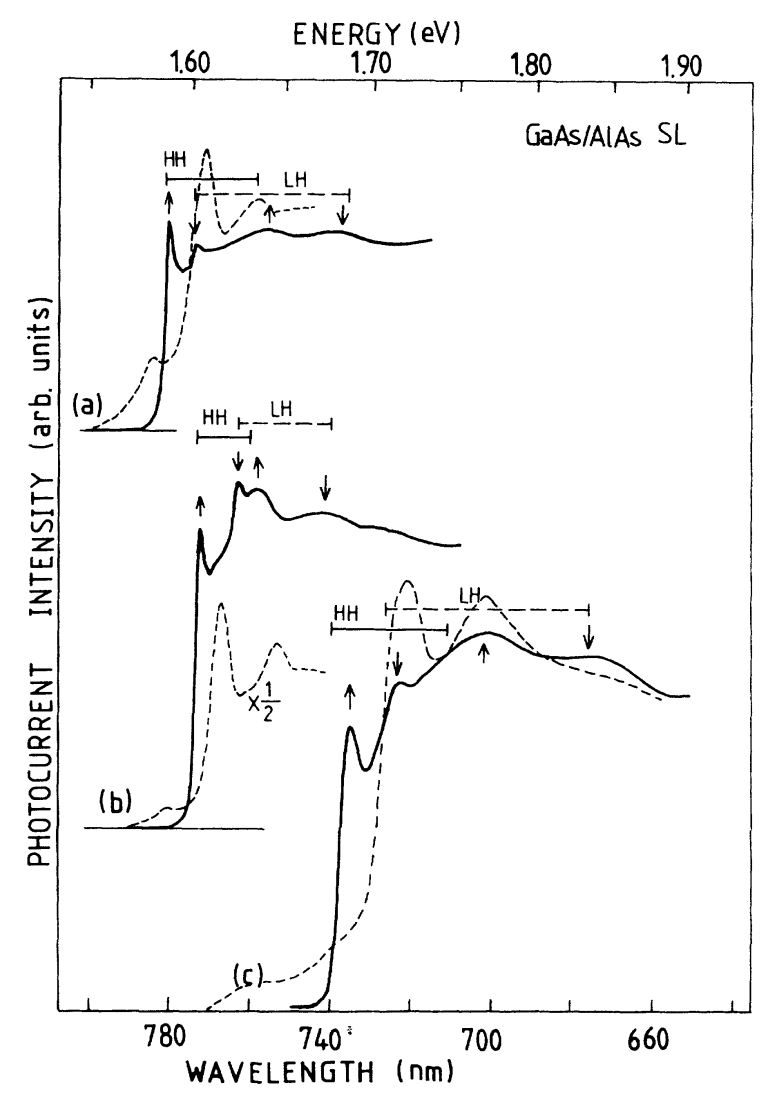

FIG. 1. Low-temperature PC spectra of three GaAs/AlAs SL diode samples with (a) $L_{Z}=62.6 \AA, L_{B}=5.7 \AA$, (b) $L_{Z}=62.6 \AA, L_{B}=8.6 \AA$, and (c) $L_{Z}=39.3 \AA, L_{B}=8.6 \AA$. The solid curves represent spectra measured near flatband condition (positive bias from +1.1 to $+1.4 \mathrm{~V}$ ), while the dashed ones were measured under perpendicular electric fields [reverse bias at $-3 \mathrm{~V}$ for (a) and (b) and at $-10 \mathrm{~V}$ for (c)]. The calculated wavelength ranges for the SL heavy-hole (light-hole) transitions are indicated by horizontal solid (dashed) bars. Upward (downward) arrows indicate prominent transitions related to heavyhole (light-hole) excitons. an upward arrow) is observed in all the spectra, indicating excitonic effects for the optical transitions. Besides the leading absorption peak, several additional peaks and/or shoulders are seen in the spectra. At first glance a second sharp peak is observed next to the leading peak which is indicated by a left downward arrow. We note that the energy separation between these two neighboring peaks increases with $L_{B}$. Broader peaks indicated by arrows on the right-hand side are located at shorter wavelengths (higher energies) and their transition energies decrease with increasing $L_{B}$. When the well width is decreased from (b) to (c), keeping the barrier thickness constant, the threshold peak is blueshifted and broader peaks are observed with wider energy separations between them. On the other hand, when an external electric field is applied along the $z$ direction, a number of new fine structures appear as shown in Fig. 1 by dashed curves. We note that in the dashed curves a main steep edge always appears at the shorter-wavelength side of the threshold peak in the corresponding flatband spectra. At the same time, the energy separation between them increases with decreasing $L_{B}$ and $L_{Z}$. The dashed spectra also contain weaker edges in the longer-wavelength region.

The experimental results depicted in Fig. 1 can rigorously be explained by miniband dispersion effects. The energy scheme for optical transitions in SL's is quite different from the QW case. When the barrier thickness is thin enough and/or the well width is narrow so that the inter-QW coupling is enhanced by tunneling, the subbands have more three-dimensional character. The bandwidth of the miniband formed along the $z$ axis is varied by choosing the $L_{B}$ and $L_{Z}$ values. In this case we have Van Hove singularities of $M_{0}$ type at the miniband bottom and of $M_{1}$ type at the upper miniband edge. When $L_{B}$ is decreased, the inter-QW coupling is enhanced, i.e., the miniband width is increased. In this case, the edge of fundamental optical absorption due to valence-toconduction subband transitions should be redshifted by the miniband dispersion effects. The experimental observation in Fig. 1 exactly corresponds to this expected behavior. This result confirms the attribution of the spectral fine structures in Fig. 1 to miniband-edge optical transitions. In order to show the effects of well and barrier parameters on the transition energies more quantitatively, the expected energies of the interband transitions are calculated using the simple Kronig-Penney model within the effective-mass approximation. ${ }^{12}$ In Fig. 1, the expected wavelength ranges for the transitions are indicated by horizontal solid (dashed) bars for the heavy-hole (light-hole) -related transitions. An excellent agreement between experimental and calculated values is observed although excitonic effects have been neglected. The two main peaks near the threshold are therefore assigned to heavy-hole (HH) and light-hole ( $\mathrm{LH}) \Gamma$ excitons associated with the miniband bottoms. We note that with decreasing $L_{B}$ the separation of the $\mathrm{HH}$ and $\mathrm{LH}$ miniband edges is reduced also in good agreement with theory. This is easily understood because the miniband character becomes more and more three-dimensional-like with decreasing $L_{B}$. 
On the other hand, the high-energy structures observed in the spectra of Fig. 1 are attributed to excitonic effects which are associated with the $M_{1}$ Van Hove singularity (II excitons). A peaked nature of the optical absorption indicates excitonic effects as origin of the $\mathrm{HH}$-related as well as LH-related transitions. We like to stress that the peaks of the high-energy $\Pi$ excitons and low-energy $\Gamma$ excitons are in excellent agreement with the calculated miniband widths. To the best of our knowledge, the peaked structures in the optical-absorption spectra represent the first clear observation of the saddle-point $\Pi$ excitons for both $\mathrm{HH}$ and $\mathrm{LH}$ excitons. Although not shown here, excellent agreement is also obtained between the experimental and theoretical transition energies for $\Gamma$ and $\Pi$ excitons over a wide range of well and barrier parameters.

Further evidence for our assignment of the minibandedge optical transitions comes from investigations of the electric-field effects. Although not discussed in detail here (since it is outside the scope of this paper; see Ref. 13 , for example), the results of the external-field effects in Fig. 1 are well explained in terms of Wannier-Stark localization. The weaker edge at longer wavelength observed in the dashed spectra of Fig. 1 is attributed to the spatially indirect first-order Stark-ladder transition. The applied electric field is high enough to generate zeroth order Stark-ladder (i.e., QW interwell direct) transitions, but not strong enough to create the quantum confined Stark effect (which causes redshifts of the QW exciton peak). ${ }^{14}$ Hence, the QW transitions should be located near the middle of the optical-transition miniband widths. Our experimental observations in Fig. 1 directly prove this for both $\mathrm{HH}$ and LH transitions. Careful comparison of the solid and dashed spectra in Fig. 1 reveals that the line shape of SL $\Gamma$ excitons is much sharper than that of QW excitons in all three samples. The linewidth narrowing of the SL $\Gamma$ excitons is also ascribed to miniband dispersion effects which average out the interface potential fluctuations over the length scale of the SL exciton wave function along the $z$ direction. ${ }^{15}$ Thus, the energy position and the linewidth of the optical transitions are all consistent with our assumption of miniband dispersion effects on the fundamental absorption spectra.

The PC signals of SL $\Gamma \mathrm{HH}$ excitons depicted in Fig. 1 exhibit a considerable reduction of the peak intensity when the miniband width becomes narrower (sample $b$ ). This trend is also true for narrower wells (sample $c$ ). These PC spectral features reflect the PC-generation mechanism which is based on optical-absorption and carrier-transport processes. The reduced PC intensities result from competitive exciton recombination channels for photogenerated carriers to be collected by the electrodes. This tendency is found to be enhanced in those SL samples with narrower minibands. We assume that the radiative recombination lifetime becomes shorter as the SL exciton shows more and more two dimensional characters. Therefore, it is better to use PLE for the investigation of the sample with narrow miniband widths. The PLE results obtained from two monolayer-controlled $(\mathrm{GaAs})_{24}(\mathrm{AlAs})_{n}$ SL samples are shown in Fig. 2. In the PLE spectrum (a) from the $n=4$ SL sample, distinct

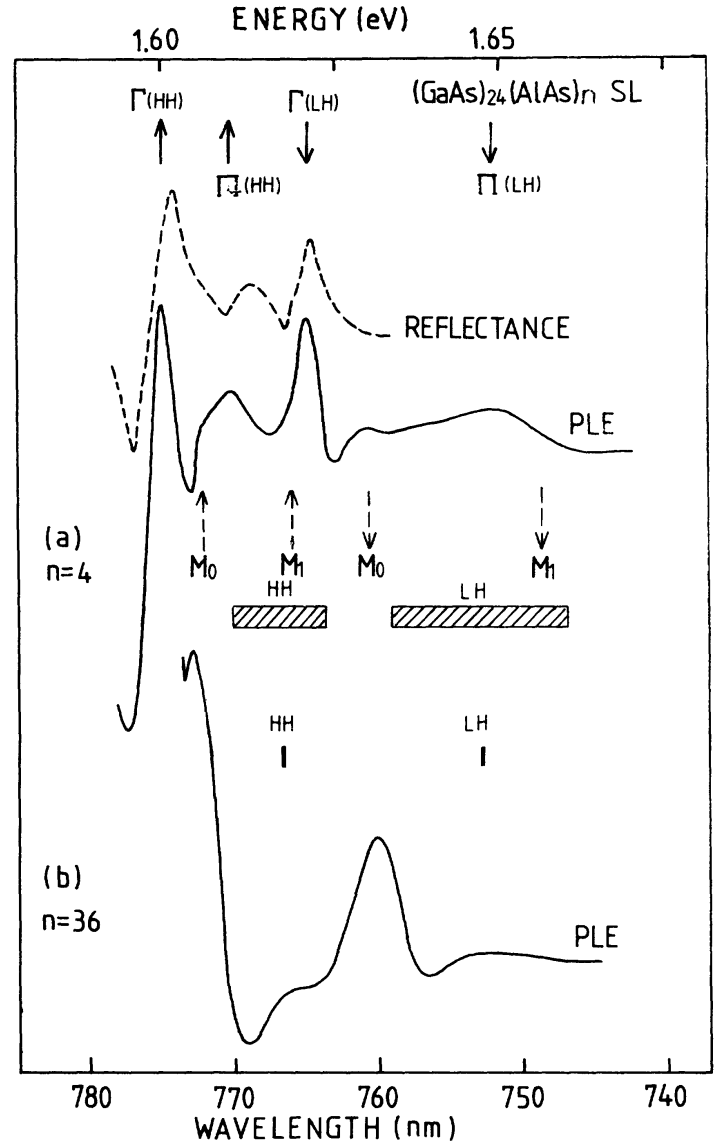

FIG. 2. PLE spectra (solid curves) of monolayer-controlled $(\mathrm{GaAs})_{24}(\mathrm{AlAs})_{n}$ SL samples. Spectrum (a) is from the $n=4 \mathrm{SL}$ sample and (b) from the $n=36 \mathrm{QW}$ sample. The calculated wavelength ranges for the heavy-hole (HH) and light-hole (LH) transitions are indicated. Upward (downward) solid arrows indicate prominent transitions related to heavy-hole (light-hole) excitons, the dashed ones to band-to-band transitions. The reflectance spectrum (dashed curve) is taken from sample (a).

structures are clearly observed and easily identified, as indicated in the figure, while the spectrum observed from the $n=36 \mathrm{SL}$ sample (b) is consistent with previous studies. ${ }^{8,9}$ Strong $\mathrm{HH}$ and $\mathrm{LH}$ exciton resonances are located below the continuum edges in spectrum (b). When we compare the measured and calculated subband energy positions, the observed fine structures in the absorption spectrum (a) are all well explained by the miniband-edge singularities and their excitonic effects. This means that four enhanced excitonic features due to $\Gamma(\mathrm{HH}), \Pi(\mathrm{HH})$, $\Gamma(\mathbf{L H})$, and $\Pi(\mathbf{L H})$ transitions are clearly observed. In addition, the distinct miniband edges at the $M_{0}$ singular points are also identified for both $\mathrm{HH}$ and LH. Based on these results, we estimate changes of the $\mathrm{HH}$ exciton binding energies from the $\mathrm{QW}(\sim 12 \mathrm{meV})$ to the $\mathrm{SL}(\sim 6$ $\mathrm{meV}$ ). In order to further evidence the Coulomb effects on the PLE spectra, a reflectance spectrum is also shown in Fig. 2. An enhanced oscillator strength is clearly observed for the $\Pi(\mathbf{H H})$ transition which supports our assignment. Finally, it is noteworthy that the line-shape asymmetry with a steep decrease on the high-energy side 
of the $\Gamma$ (LH) transition may be due to the $M_{1}$ singularity for the $\mathrm{HH}$ upper edge. Considering the miniband dispersion calculations, there exist some minor deviations for the measured transition energies. These might be caused by interface-potential and layer-thickness fluctuation effects.

In summary, we have investigated band-edge optical transitions in GaAs/AlAs superlattices with different miniband widths by low-temperature photocurrent (PC), photoluminescence-excitation and reflectance spectroscopy. By virtue of systematic sample parameter variations, distinct transition peaks are observed which are assigned to the $M_{0^{-}}$and $M_{1}$-type Van Hove singularities at the lower and upper miniband edges. The involvement of the excitonic effects is demonstrated for both heavy-hole- and light-hole-related transitions. An excellent coincidence is obtained between the observed and calculated optical transition energies, using a simple Kronig-Penney model.

This work was supported in part by Scientific Research Grant-in-Aid No. 05044109 from the Ministry of Education, Science and Culture of Japan.
${ }^{1}$ J. Bleuse, G. Bastard, and P. Voisin, Phys. Rev. Lett. 60, 220 (1988).

${ }^{2}$ E. E. Mendez, F. Agullo-Rueda, and J. M. Hong, Phys. Rev. Lett. 60, 2426 (1988).

${ }^{3}$ H. Schneider, K. Kawashima, and K. Fujiwara, Phys. Rev. B 44, 5943 (1991).

${ }^{4}$ M. Nakayama, I. Tanaka, H. Nishimura, K. Kawashima, and K. Fujiwara, Phys. Rev. B 44, 5935 (1991).

${ }^{5}$ K. H. Schmidt, A. D'Intino, N. Linder, M. Tuna, G. H. Döhler, H. T. Grahn, K. Kawashima, and K. Fujiwara, Superlatt. Microstruct. 12, 181 (1992).

${ }^{6}$ L. Esaki and T. Tsu, IBM J. Res. Dev. 14, 61 (1970).

${ }^{7}$ H. Chu and Y.-C. Chang, Phys. Rev. B 36, 2946 (1987).

${ }^{8}$ J. J. Song, P. S. Jung, Y. S. Yoon, H. Chu, and Y.-C. Chang, Phys. Rev. B 39, 5562 (1989).
${ }^{9}$ B. Deveaud, A. Chomette, F. Clerot, A. Regreny, J. C. Maan, R. Romestain, G. Bastard, H. Chu, and Y.-C. Chang, Phys. Rev. B 40, 5802 (1989).

${ }^{10}$ E. O. Kane, Phys. Rev. 180, 852 (1969).

${ }^{11}$ K. Kawashima, K. Fujiwara, T. Yamamoto, M. Sigeta, and K. Kobayashi, Surf. Sci. 267, 643 (1992).

${ }^{12}$ K. Fujiwara, R. Cingolani, and K. Ploog, Solid State Commun. 72, 389 (1989).

${ }^{13}$ K. Kawashima, T. Yamamoto, K. Kobayashi, and K. Fujiwara, Phys. Rev. B 47, 9921 (1993).

${ }^{14}$ D. A. B. Miller, J. S. Weiner, and D. S. Chemla, IEEE J. Quantum Electron. QE-22, 1816 (1986).

${ }^{15}$ P. S. Jung, J. M. Jacob, J. J. Song. Y.-C. Chang, and C. W. Tu, Phys. Rev. B 40, 6454 (1989). 